Proceedings of ASME Turbo Expo 2021

Turbomachinery Technical Conference and Exposition

June 7 - 11, 2021, Virtual, Online

GT2021-58477

\title{
A DISCRETE ELEMENT METHODS-BASED MODEL FOR PARTICULATE DEPOSITION AND REBOUND IN GAS TURBINES
}

\author{
Jack G. Gaskell; Matthew McGilvray \& David R. H. Gillespie \\ Oxford Thermofluids Institute \\ Department of Engineering Science \\ University of Oxford \\ Oxford, OX2 0ES, UK \\ Email: jack.gaskell@eng.ox.ac.uk
}

\begin{abstract}
The secondary air system and cooling passages of gas turbine components are prone to blockage from sand and dust. Prediction of deposition requires accurate models of particle transport and thermo-mechanical interaction with walls. Bounce stick models predict whether a particle will bounce, stick, or shatter upon impact and calculate rebound trajectories if applicable. This paper proposes an explicit bounce stick model that uses analytical solutions of adhesion, plastic deformation and viscoelasticity to time-resolve collision physics. The Discrete-Element Methods (DEM) model shows good agreement when compared to experimental studies of micron and millimetre-scale particle collisions, requiring minimal parametric fitting. Non-physical values mechanical properties, artifices of previous models, are thus eliminated. Further comparison is made to the best resolved and industry standard semi-empirical models available in literature. In addition to coefficients of restitution, other variables crucial to accurately model rebound, for example angular velocity, are predicted. The time-stepping explicit approach allows full coupling between internal processes during contact, and shows that particle deformation and hence viscoelasticity play a significant role in adhesion. Modelling time-dependent internal variables such as wall-normal force create functionality for future modelling of arbitrarily shaped particles, the physics of which has been shown by previous work to differ significantly from that of spheres. To date these effects have not been captured well using by higher-level energy-based models.
\end{abstract}

\footnotetext{
${ }^{*}$ Address all correspondence to this author.
}

\section{NOMENCLATURE}

$\begin{array}{ll}\text { Abbreviations } \\ \text { ARD } & \text { Arizona Road Dust } \\ \text { CV } & \text { Critical Velocity } \\ \text { DEM } & \text { Discrete Element Methods } \\ \text { JKR } & \text { Johnson, Kendall and Roberts } \\ \text { OSU } & \text { Ohio State University } \\ \text { TN } & \text { Thornton and Ning } \\ \text { Roman } & \text { Symbols } \\ a & \text { Contact radius, } \mathrm{m} \\ a_{0} & \text { Equilibrium contact radius, } \mathrm{m} \\ a_{\mathrm{c}} & \text { Critical pull-off contact radius, } \mathrm{m} \\ E & \quad \text { Young's modulus, Pa } \\ F_{\mathrm{c}} & \text { Critical elastic pull-off force, } \mathrm{N} \\ F_{\mathrm{f}} & \text { Tangential frictional force, } \mathrm{N} \\ F_{\mathrm{n} 1} & \text { Equivalent elastic force for the same contact radius, } \mathrm{N} \\ F_{\mathrm{nd}} & \text { Viscoelastic normal dissipative force, } \mathrm{N} \\ G & \text { Shear modulus, Pa } \\ I & \text { Moment of inertia, kg } \mathrm{m}^{2} \\ K & \text { Particle stiffness coefficient from TN model, Nm }{ }^{-1} \\ k & \text { Stiffness coefficient used in viscoelastic deformation or } \\ & \text { tangential contact patch deformation, } \mathrm{Nm}^{-1} \\ M_{\mathrm{f}} & \text { Moment due to friction, Nm } \\ M_{\mathrm{r}} & \text { Moment due to rolling adhesion, Nm } \\ p_{\mathrm{y}} & \text { Limiting surface contact pressure, Pa } \\ R & \text { Particle radius, } \mathrm{m} \\ V & \text { Velocity, ms } \\ x_{0} & \text { Characteristic thickness of particle-wall squeeze-film, m }\end{array}$




\section{Greek Symbols}

$\delta_{\text {gap }} \quad$ Minimum particle-wall separation distance, $\mathrm{m}$

$\delta_{\mathrm{n}}, \delta_{\mathrm{n}} \quad$ Normal and tangential contact patch displacement, $\mathrm{m}$

$\eta \quad$ Viscoelastic dissipation coefficient, $\mathrm{kg} \mathrm{s}^{-1}$

$\gamma \quad$ Surface free energy, $\mathrm{J} \mathrm{m}^{-2}$

$\mu_{\mathrm{f}} \quad$ Fluid dynamic viscosity, Pas

$\Omega \quad$ Angular velocity, $\mathrm{rad} \mathrm{s}^{-1}$

$\rho \quad$ Particle density, $\mathrm{kg} \mathrm{m}^{-3}$

$\theta_{\mathrm{i}} \quad$ Impingement angle, ${ }^{\circ}$

$\xi \quad$ Adhesive rolling displacement, $\mathrm{m}$

\section{Non-Dimensional Parameters}

$\mathrm{CoR}_{\mathrm{n}}, \mathrm{CoR}_{\mathrm{t}}$ Normal and tangential coefficients of restitution

$e_{0} \quad$ Equivalent viscoelastic normal coefficient of restitution

$f_{\Delta \mathrm{t}} \quad$ Timestep multiplication factor

Stc Collision Stokes number

SF Squeeze-film coefficient $\delta_{\text {gap }} / x_{0}$

\section{Subscripts}

0,3 Far from the wall prior to and following collision

1,2 Immediately prior to and following collision

$\mathrm{e}, \mathrm{p}, \mathrm{u}$ Elastic, plastic and elastic-adhesive unloading phases of particle deformation

n Normal

r Rebound

$\mathrm{t}$ Tangential

\section{Superscripts}

* Composite material property or value at the point of maximum deformation

\section{INTRODUCTION}

Ingested atmospheric contaminants are known to cause significant damage to gas turbine components through deposition, erosion and corrosion [1]. Upon ingestion, large particles erode compressor aerofoils and hence increase the required design surge margin, limiting whole engine performance. Smaller particles and fragments from these earlier collisions are then transported to the turbine and combustor where secondary air systems and cooling passages are prone to blockage throughout fleet service life. This drives significant increases in maintenance costs and risks catastrophic failure of hot gas path components. Therefore, the ability to accurately model deposition is desirable to quantify cost of ownership effects and to enable the design of more durable components. Bounce stick models predict whether a particle will bounce, stick or shatter upon collision with a wall and hence are crucial to accurately modelling deposition and transport. The predicted rebound trajectories are equally important to the binary bounce/stick criterion as exposure to different flowfields, particularly in the near wall region, will alter the likelihood and location of subsequent collision and deposition. Comparisons between numerical simulations of particle deposition and experimental observations have shown that while first bounces are well predicted by tracking models, rebound affects the spatial distribution of downstream deposition [2]. Collisions are defined as in Figure 1 with particle rebound trajectories described through normal and tangential coefficients of restitution, $\mathrm{CoR}_{\mathrm{n}}$ and $\mathrm{CoR}_{\mathrm{t}}$, defined in Equation 1:

$$
\mathrm{CoR}_{\mathrm{i}}=\left|\frac{V_{\mathrm{i} 3}}{V_{\mathrm{i} 0}}\right|
$$

where $\mathrm{CoR}_{\mathrm{n}}=0$ implies the particle has stuck. Subscript zero describes the velocity component shortly before collision with the wall, while subscript three describes the velocity shortly after last contact as described by the explanation of squeeze-film damping in the Methodology section. Airborne particulates near ground level are predominantly comprised of aluminosilicates [3] and are non-spherical, with aspect ratios between 1.6-1.9 [3,4]. The size of particulates ingested by the engine is not necessarily representative of those present in the secondary air system as they are often shattered by collisions with compressor blades and larger particles are centrifuged away from compressor bleeds. Therefore, particles at the compressor exit have an average diameter of $6 \mu \mathrm{m}$ [5]. Existing bounce stick models, notably [6-8] assume that particles are cylindrical/spherical to reduce mathematical complexity while taking a high-level approach to modelling, typically defining collision dynamics in terms of kinetic energy losses. While these approaches have been shown to replicate experimental results, the degree of parametric fitting required to do so implies that not all aspects of the collision physics are being accurately modelled. The necessity of fitting to experimental data also limits their use in industry-relevant simulations which consider a range of particle sizes, shapes and compositions. Existing models have also neglected collision-induced rotation (CIR), which was shown by [9] to have a significant effect on micronscale particle rebound trajectories through the Magnus force. It should be noted that while rotation is predicted by [8], the relevance to particle trajectory is not discussed and the results not validated independently of total $\mathrm{CoR}$. Particle non-sphericity was shown by $[10,11]$ to affect particle rebound trajectory and angular velocity, likely also coupling with Magnus force effects. Some models apply stochastic corrections to account for non-sphericity [11], providing accurate rebound predictions, however it should be noted that this is necessarily limited to the specific particle materials, sizes and shapes tested. Applying stochastic corrections to the overall rebound trajectories also does not allow accurate physical modelling of the collision physics, which is a focus of this paper.

Adhesion through van der Waals forces has been shown by $[6,12]$ to significantly reduce particle $\mathrm{CoR}_{\mathrm{n}}$ for diameters less than $10 \mu \mathrm{m}$, particularly where collision normal velocities 
are low. Other factors that affect particle rebound, also modelled by [6], are plastic deformation and friction with the substrate. Viscoelastic losses have generally not been modelled by previous work, although discussion of the critical velocity model in [7] recognises their importance to predicting $\mathrm{CoR}_{\mathrm{n}}$. It is important to consider not only solid-phase viscoelastic dissipation but also that of the fluid: squeeze-film damping between the particle and surface was shown analytically by $[13,14]$ to reduce $\mathrm{CoR}_{\mathrm{n}}$ towards zero for impacts where both the particle-surface separation gaps and Stokes numbers are small. This is supported by experimental evidence [15] where spheres with $R e<10$ just before impact failed to rebound, without significant adhesive effects.

\section{Discrete Element Methods}

This paper describes an analytically-explicit bounce stick model based on Discrete Element Methods (DEM). DEM codes are often used to simulate the behaviour of granular assemblies with large numbers of particles, originally developed for geotechnical purposes by $[16,17]$. The present work will include the effects of adhesion, plastic deformation, friction and viscoelasticity to predict $\mathrm{CoR}_{\mathrm{n}}, \mathrm{CoR}_{\mathrm{t}}$ and angular velocity, $\Omega$. It differs from most DEM implementations as it only considers single particle collisions between the point of impact with and rebound from a flat, smooth substrate.

The DEM-based approach resolves all relevant collision physics throughout the contact time and hence allows coupling between internal variables that is neglected by high-level energy-based models. Particles are assumed to be spherical however it is noted that the DEM approach is well-suited for extensions that model non-sphericity due to its force-based approach. Indeed, it is simple to include any phenomenon that can be expressed in the force domain where full coupling with other internal parameters is guaranteed by the explicit process. This is a key benefit of the DEM approach over energy-based models. In further work, it is intended that this model will be used with commercial CFD solvers to predict deposition in gas turbine components. Due to the computational cost per particle impact, as described below, it is proposed that a machine learning-based surrogate model is trained on the outputs of the DEM code to predict rebound trajectories at a fraction of the computational cost of direct implementation in CFD. Prototypes of this approach produce promising results which will be presented by the authors in future work that describes the full workflow of integrating the DEM approach in commercial CFD solvers. These approaches are intended to have similar computational cost to existing bounce stick models and hence should allow deposition to be simulated in OEM components with $\mathscr{O}\left(10^{5}\right)$ particles injected. The consequent ability to model spatially resolved deposition will allow OEMs to design

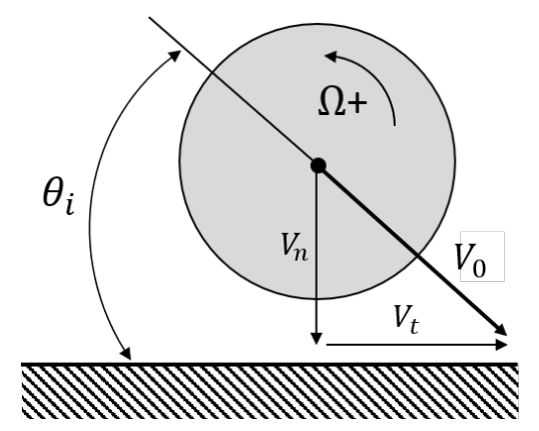

FIGURE 1. PARTICLE SIGN CONVENTION

deposition-tolerant hardware, prolonging the life of hot gas path components.

\section{METHODOLOGY}

Discrete Element models are explicit time-stepping schemes that follow the interactions and transport of individual particles. By modelling the forces that act on each particle during the contact time, the equations of motion are integrated and hence the velocities and displacements during collisions can be found.

When modelling deposition in real components, external factors like gas temperature $[18,19]$ and surface roughness $[20,21]$ are known to affect deposition rate. Although neither is included in the present work, both factors are treated as external to the model described here and hence can be included by modifying the input parameters. Where the scale of surface roughness is greater than the particle radii, surface roughness can be accounted for using an stochastically augmented angle of incidence described by a modified normal distribution [21]. Similarly, assuming the particle and surface materials to be homogeneous and isotropic, mechanical properties can be modified to account for temperature as in $[6,8]$. This would include thermochemical effects and phase changes. Post-deposition effects like sintering are out of scope for the present work and should be included in a suitable deposit evolution model. Similarly, erosion and particle fragmentation are not included here hence limiting the scope of the current DEM approach to ductile impacts like those most commonly found downstream of the compressor. However, deformation of the wall is accounted for through the use of composite elastic moduli like $E^{*}$ and $G^{*}$, and taking the lower of the yield stresses of the particle and substrate material as an input to the simulation.

When modelling particle motion, it is assumed that the only angular velocity vector is about the axis normal to the page in Figure 1 , hence all velocity vectors exist in the plane normal to the wall. The particle equations of motion are shown in Equations 2 and 3: 


$$
\begin{gathered}
m \frac{d \bar{V}}{d t}=\left[\begin{array}{c}
m \frac{d V_{\mathrm{f}}}{d t} \\
m \frac{d V_{\mathrm{n}}}{d t} \\
0
\end{array}\right]=\left[\begin{array}{c}
F_{\mathrm{f}} \\
F_{\mathrm{n}}+F_{\mathrm{nd}} \\
0
\end{array}\right] \\
I \frac{d \Omega}{d t}=M_{\mathrm{f}}+M_{\mathrm{r}}
\end{gathered}
$$

where $m$ is the particle mass, $F_{\mathrm{f}}$ is the effective frictional force that acts on the particle, $F_{\mathrm{n}}$ is the normal force that includes elastic and plastic deformation, and adhesive effects, while $F_{\text {nd }}$ is the normal dissipative force resulting from viscoelasticity. In Equation 3, $M_{\mathrm{f}}$ is the moment resulting from effective friction with the surface $\left(R F_{\mathrm{f}}\right)$ and $M_{\mathrm{r}}$ is the moment resulting from adhesion while rolling. The simulation timestep $\Delta t$ is calculated using the particle elastic response time $T_{\mathrm{c}}$, as in [22]. The elastic response time, an order of magnitude estimation of the total contact time, is multiplied by a factor $f_{\Delta \mathrm{t}}$ that is much less than unity to ensure particle deformation throughout the contact time is adequately resolved. In effect, this makes the value of $f_{\Delta \mathrm{t}}$ approximately equal to the number of time steps. A series of DEM collision simulations were performed for a generic adhesive, elastic perfectly-plastic microsphere with varying $f_{\Delta \mathrm{t}}$ to study timestep sensitivity. These results are shown in Figure 2 where $f_{\Delta \mathrm{t}}=1 \times 10^{-4}$ was deemed the most computationally efficient with a runtime of $8.5 \mathrm{~s}$ per impact. This compares to a runtime of $1100 \mathrm{~s}$ for $f_{\Delta \mathrm{t}}=1 \times 10^{-5}$ running on a 10 core PC with 64GB RAM.

$$
\Delta t=f_{\Delta \mathrm{t}} T_{\mathrm{c}}=f_{\Delta \mathrm{t}} 2 R\left(\frac{\rho^{2}}{V_{\mathrm{i}} E_{\mathrm{p}}^{2}}\right)^{\frac{1}{5}}
$$

Note that $E_{\mathrm{p}}$ is the particle Young's modulus. Tangential force is dependent on normal force through friction, however the normal force has no such dependency on the tangential. Therefore, the normal force is calculated first during each time step. In most DEM implementations, tangential and normal models are chosen independently of one another and therefore they are presented separately in the following subsections.

\section{Normal Force Model}

The normal force model derived by Thornton and Ning [23], herein referred to as the TN model, is used to calculate the normal force considering adhesion and plastic deformation while the

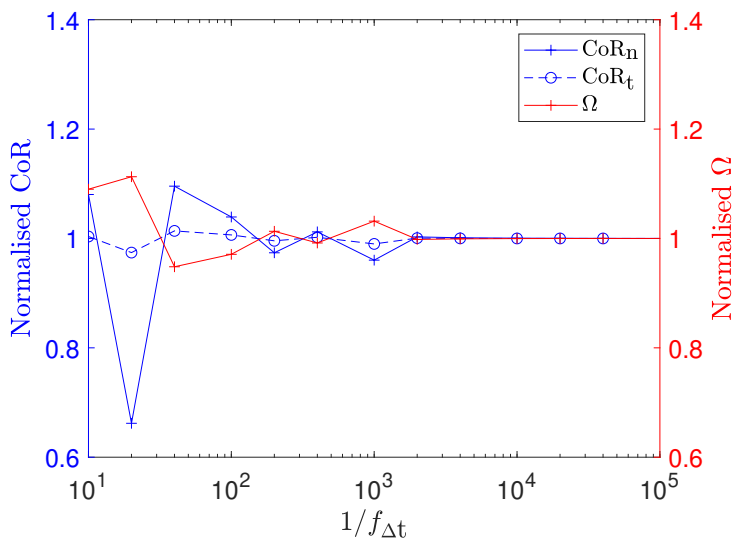

FIGURE 2. SENSITIVITY OF $\mathrm{CoR}_{\mathrm{n}}, \mathrm{COR}_{\mathrm{t}} \mathrm{AND} \Omega$ TO VARYING TIMESTEPS. RESULTS ARE NORMALISED BY THE VALUE FROM THE MINIMUM TIMESTEP.

normal dissipative force, accounting for solid-phase viscoelastic effects, is calculated using the model presented in [22]. Squeezefilm effects are modelled using the approach developed in [14], described at the end of this subsection. Adhesion is based on JKR theory [24] which is widely used for modelling the adhesive behaviour of micron-scale compliant particles. The TN model divides the contact time of an elastic perfectly-plastic adhesive particle into three distinct stages: elastic-adhesive compression; plastic-adhesive compression and elastic-adhesive unloading. These correspond to points 1-2, 2-3 and 3-5 in the normal force vs. normal overlap graph in Figure 3, where arrows show the direction of loading and unloading. Depending on the stage, a different normal stiffness coefficient is calculated and multiplied by the normal displacement at that timestep to obtain the incremental normal force. Throughout the contact time, in every stage, the equivalent elastic normal force at the contact patch is calculated:

$$
\begin{aligned}
F_{\mathrm{n}, \mathrm{el}}(t)=F_{\mathrm{n}, \mathrm{el}}(t-\Delta t) & +\Delta F_{\mathrm{n}, \mathrm{el}} \\
= & F_{\mathrm{n}, \mathrm{el}}(t-\Delta t)+K_{\mathrm{ne}}(t) V_{\mathrm{n}}(t-\Delta t) \Delta t
\end{aligned}
$$

where the elastic normal contact stiffness $K_{\text {ne }}$ is a function of the elastic critical pull-off force $F_{\mathrm{c}}$ and the equivalent Hertzian normal force $F_{\mathrm{n} 1}$ that would produce the same contact radius $a$ without adhesion:

$$
K_{\mathrm{ne}}(t)=2 E^{*} a(t)\left[\frac{3 \sqrt{F_{\mathrm{n} 1}(t)}-3 \sqrt{F_{\mathrm{c}}}}{3 \sqrt{F_{\mathrm{n} 1}(t)}-\sqrt{F_{\mathrm{c}}}}\right]
$$




$$
\begin{gathered}
F_{\mathrm{c}}=\frac{3}{2} \pi \gamma R \\
F_{\mathrm{n} 1}(t)=F_{\mathrm{n}, \mathrm{el}}(t-\Delta t)+2 F_{\mathrm{c}} \pm \sqrt{4 F_{\mathrm{n}, \mathrm{el}}(t-\Delta t) F_{\mathrm{c}}+4 F_{\mathrm{c}}^{2}} \\
a(t)=\left[\frac{3 R F_{\mathrm{n} 1}(t)}{4 E^{*}}\right]^{\frac{1}{3}}
\end{gathered}
$$

Note that $R$ is the particle radius, $\gamma$ is the surface free energy and $E^{*}$ is the composite Young's modulus of the particle and surface materials. The final term in Equation 8 is initially positive, switching to negative once $F_{\mathrm{n}}(t)$ (Equations 5, 14 and 17) is less than or equal to $-F_{\text {cr }}$ when unloading (Equations 7 and 15). This is shown by point 4 in Figure 3 . At the start of contact, the contact radius is less than the value at yielding, $a_{\mathrm{y}}$ (between points 1 and 2 in Figure 3). Therefore, the particle undergoes elasticadhesive compression and the actual normal force at contact $F_{\mathrm{n}}$ is equal to the equivalent elastic value $F_{\mathrm{n}, \mathrm{el}}$ :

$$
F_{\mathrm{n}}(t)=F_{\mathrm{n}}(t-\Delta t)+\Delta F_{\mathrm{n}, \mathrm{el}}
$$

As calculating $a_{\mathrm{y}}$ directly from Equation 11 is complex, the contact radius at yielding $a_{\mathrm{y}}$ is found using a lookup table. Outside of the time-stepping loop, Equation 11 is solved for a large number of possible values of $a_{\mathrm{y}}$, linking a range of yield stresses $\left(\sigma_{\mathrm{y}}\right)$ to corresponding yield radii. Using 1D interpolation, a suitable $a_{\mathrm{y}}$ is found using the lower of the yield stresses of the particle and substrate which are inputs to the model. Note that the limiting contact pressure $p_{\mathrm{y}}$ is reached at 2.4 times $\sigma_{\mathrm{y}}[25]$.

$$
p_{\mathrm{y}}=2.4 \sigma_{\mathrm{y}}=\frac{2 E^{*} a_{\mathrm{y}}}{\pi R}-\left(\frac{2 \gamma E^{*}}{\pi a_{\mathrm{y}}}\right)^{\frac{1}{2}}
$$

Where the contact radius $a$ is greater than $a_{\mathrm{y}}$, yielding occurs (point 2 in Figure 3) and the particle undergoes plastic-adhesive compression between points 2 and 3 . Note that the actual normal force $F_{\mathrm{n}}$ and equivalent elastic normal force $F_{\mathrm{n} \text {,el }}$ diverge here, with $F_{\mathrm{n}}$ proceeding on a tangent to the $F_{\mathrm{n} \text {,el }}$ curve at point 2 . To achieve this, a new normal stiffness $K_{\mathrm{np}}$ is calculated using the

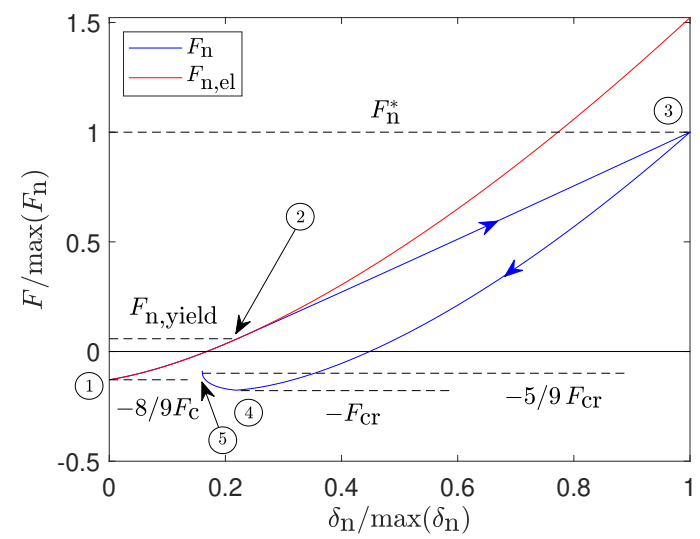

FIGURE 3. NORMALISED NORMAL FORCE VS. NORMAL OVERLAP FOR THE REBOUND OF AN ADHESIVE ELASTIC PERFECTLY-PLASTIC MICROSPHERE. ARROWS SHOW THE DIRECTION OF COMPRESSION AND UNLOADING.

critical contact radius $a_{\mathrm{c}}$, [23], defined as the contact radius $a$ where $F_{\mathrm{n}}=-F_{\mathrm{c}}$ :

$$
K_{\mathrm{np}}(t)=3 \pi R p_{\mathrm{y}}-\frac{2 a_{\mathrm{y}} E^{*}\left[a_{\mathrm{c}} / a(t)\right]^{3 / 2}}{3-\left[a_{\mathrm{c}} / a(t)\right]^{3 / 2}}
$$

$$
a_{\mathrm{c}}=\left(\frac{9 \pi \gamma R^{2}}{8 E^{*}}\right)^{\frac{1}{3}}
$$

Therefore, the new normal contact force is calculated:

$$
\begin{aligned}
F_{\mathrm{n}}(t)=F_{\mathrm{n}}(t-\Delta t)+ & \Delta F_{\mathrm{np}} \\
& =F_{\mathrm{n}}(t-\Delta t)+K_{\mathrm{np}}(t) V_{\mathrm{n}}(t-\Delta t) \Delta t
\end{aligned}
$$

Once the particle normal velocity changes sign at the point of maximum penetration, point 3 in Figure 3, it enters the elasticadhesive unloading regime between points 3 and 5. If plastic deformation occurs, the contact patch is flattened and hence its effective radius of curvature is increased during unloading. To account for this, a new particle radius $R_{\mathrm{p}}$ is used in place of $R$ in the equations that govern deformation: Equations 7, 8 and 9 to produce $F_{\mathrm{cr}}, F_{\mathrm{n} 1 \mathrm{r}}$ and subsequent values of $a$.

$$
R_{\mathrm{p}}=\frac{R F_{\mathrm{n} 1}^{*}}{F_{\mathrm{n}}^{*}+\sqrt{4 F_{\mathrm{c}} F_{\mathrm{n} 1}^{*}}}
$$


Note that $F_{\mathrm{n} 1}^{*}$ and $F_{\mathrm{n}}^{*}$ denote values at the point of maximum penetration, with the latter shown in Figure 3. These values are used to calculate a new contact stiffness $K_{\text {nu }}$ and hence the normal force at the contact patch between points 3 and 5:

$$
\begin{gathered}
K_{\mathrm{nu}}(t)=2 E^{*} a(t)\left[\frac{3 \sqrt{F_{\mathrm{n} 1 \mathrm{r}}(t)}-3 \sqrt{F_{\mathrm{cr}}}}{3 \sqrt{F_{\mathrm{n} 1 \mathrm{r}}(t)}-\sqrt{F_{\mathrm{cr}}}}\right] \\
F_{\mathrm{n}}(t)=F_{\mathrm{n}}(t-\Delta t)+\Delta F_{\mathrm{nu}} \\
=F_{\mathrm{n}}(t-\Delta t)+K_{\mathrm{nu}}(t) V_{\mathrm{n}}(t-\Delta t) \Delta t
\end{gathered}
$$

If yielding does not occur, contact with the surface is broken when $F_{\mathrm{n}}(t) \leq-F_{\mathrm{c}}$ and the particle will rebound along the same force-displacement curve as compression $F_{\mathrm{n}, \mathrm{el}}$. In effect, the particle stays within the elastic-adhesive compression regime throughout the contact duration and uses only Equations 5, 6 and 10 to calculate $F_{\mathrm{n}}$. If the contact patch has yielded, as in Figure 3 , the sign of \pm in Equation 8 changes when $F_{\mathrm{n}}(t) \leq F_{\mathrm{cr}}$ (point 4 ) and contact is broken when $F_{\mathrm{n}}(t) \geq-5 / 9 F_{\mathrm{cr}}$ (point 5). All force boundary conditions are taken from Table 5.1 in [26]. The increased gradient of the force-displacement curve following the point of maximum penetration $F_{\mathrm{n}}^{*}$ is due to plastic flattening of the contact patch. Unloading continues until either the particle rebounds, as in this case, or it sticks and dissipates its kinetic energy through viscoelastic dissipation while oscillating.

The normal dissipative force that accounts for solid-phase viscoelastic effects $F_{\text {nd }}$ is calculated by multiplying a dissipation coefficient $\eta_{n}$ by the particle normal velocity $V_{\mathrm{n}}$ :

$$
\begin{gathered}
\eta_{\mathrm{n}}(t)=\alpha \sqrt{m k_{\mathrm{n}}(t)} \\
k_{\mathrm{n}}(t)=\frac{4}{3} E^{*} a(t)
\end{gathered}
$$

where $\alpha$ is a sixth-order polynomial fit to an analytical solution of $e_{0}$, the $\mathrm{CoR}_{\mathrm{n}}$ if viscoelasticity were the only dissipation mechanism in a normal contact [22]. The value of $e_{0}$ is an input to the model, currently fit to experimental observations. This is the only explicit parametric fitting included in the current model. Note that the lowercase $k_{\mathrm{n}}$ is distinct from the stiffness coefficients used in the TN model. Once $F_{\mathrm{n}}$ and $F_{\text {nd }}$ are known for each time step, the normal components of the particle equations of motion in Equation 2 can be updated and the normal force can be used as an input to the tangential contact model.

Squeeze-film damping is the result of fluid motion between the particle and wall during approach and rebound, reducing the particle velocity magnitude shortly before and after the collision. As the effect primarily occurs shortly before first contact and after rebound, which define the beginning and end of the DEM timestepping loop, it is used to modify the input and output velocity vectors of the DEM as described below. Therefore, particle velocities at first and last contact with the surface are defined as $V_{\mathrm{n} 1}$ and $V_{\mathrm{n} 2}$ while values far from the wall before and after the collision are defined as $V_{\mathrm{n} 0}$ and $V_{\mathrm{n} 3}$. Therefore, $V_{\mathrm{n} 0}$ and $V_{\mathrm{n} 3}$ are the input and output velocity vectors to the simulation while $V_{\mathrm{n} 1}$ and $V_{\mathrm{n} 2}$ are effectively internal variables. The magnitude of the squeeze-film effect depends on the collision Stokes number Stc:

$$
\text { Stc }=\frac{2 \rho V_{\mathrm{n} 0} R}{9 \mu_{\mathrm{f}}}
$$

where $\mu_{\mathrm{f}}$ is the fluid dynamic viscosity. A smaller value of Stc implies greater influence of fluid viscosity on particle motion and hence a greater reduction in velocity. Expressions that relate $V_{\mathrm{n} 0}$ to $V_{\mathrm{n} 1}$ and $V_{\mathrm{n} 2}$ to $V_{\mathrm{n} 3}$ were derived by [14]:

$$
\begin{gathered}
\frac{V_{\mathrm{n} 1}}{V_{\mathrm{n} 0}}=1+\frac{1}{S t c} \ln \left(\delta_{\mathrm{gap}} / x_{0}\right) \\
\frac{V_{\mathrm{n} 2}-V_{\mathrm{n} 3}}{V_{\mathrm{n} 0}}=\frac{1}{S t c} \ln \left(\delta_{\mathrm{gap}} / x_{0}\right)
\end{gathered}
$$

where $\delta_{\text {gap }}$ is the minimum separation distance, which for practical purposes is surface roughness, and $x_{0}$ is the squeeze-film thickness which is generally equal to $0.01 R$ [27].

\section{Tangential Model}

The tangential forces that act at the contact patch are calculated using the widely-used model proposed by Cundall and Strack [17] with additional equations from [28] to account for rolling adhesion. The slip velocity between the particle and surface $V_{\mathrm{s}}$ is key to the direction of frictional force. Here, the slip velocity will initially be positive where the frictional force is initially negative:

$$
V_{\mathrm{s}}(t)=V_{\mathrm{t}}(t)+R \Omega(t)
$$


noting that angular velocity $\Omega$ is negative in the clockwise direction, hence ensuring $V_{\mathrm{s}}<V_{\mathrm{t}}$. Cundall and Strack's model incorporates a spring and dashpot to model tangential deformation at the contact patch with a slider to account for frictional effects. Therefore, the tangential force at the contact patch $F_{\mathrm{f}}$ is calculated using Equations 24 and 25:

$$
\begin{gathered}
F_{\mathrm{f}}(t)=\min \left[\left|k_{\mathrm{t}}(t) \delta_{\mathrm{t}}(t)+\eta_{\mathrm{t}} V_{\mathrm{s}}(t)\right|,-\mu\left|F_{\mathrm{n}}(t)+2 F_{\mathrm{c}}\right|\right] \\
\delta_{\mathrm{t}}(t)=\delta_{\mathrm{t}}(t-\Delta t)-V_{\mathrm{s}}(t) \Delta t
\end{gathered}
$$

where $k_{\mathrm{t}}(t)=8 G^{*} a(t)$ is the tangential stiffness, $\mu$ is the coefficient of friction and $\eta_{\mathrm{t}}=\eta_{\mathrm{n}}$. The rolling adhesion model from Dominik and Tielens [28] uses a rolling displacement $\xi$ as defined below to calculate the moment due to adhesion that opposes the particle's angular velocity. This moment is due to an asymmetrical pressure distribution between the front and rear of a rolling particle as points at the rear "jump-off" the surface at a higher normal overlap than those at the front "jump-on". Therefore, when rolling the contact patch lags behind the centroid and the rolling displacement is the tangential projection of the distance between the centre of the contact patch and the particle centroid. Below a critical value $\xi_{\text {crit }}$, the contact patch remains stuck. Once $\xi=\xi_{\text {crit }}$, the contact patch begins to move at the same tangential velocity as the centroid, lagging $\xi_{\text {crit }}$ behind. Clearly, there is coupling between $\xi$ and the tangential contact patch deformation $\delta_{\mathrm{t}}$ in Equation 24. However, to reduce complexity it is assumed that the two models work independently with $\xi$ defined as the time integral of the rolling velocity $V_{\mathrm{L}}=R \Omega$, limited in magnitude to $\xi_{\text {crit }}$. Therefore, the moment due to adhesive rolling $M_{\mathrm{r}}$ is given by:

$$
\begin{gathered}
M_{\mathrm{r}}(t)=-4 F_{\mathrm{c}} \xi(t)\left(\frac{a(t)}{a_{0}}\right)^{\frac{3}{2}} \\
a_{0}=\left(\frac{9 \pi \gamma R^{2}}{E^{*}}\right)^{\frac{1}{3}}
\end{gathered}
$$

where $a_{0}$ is the equilibrium contact radius. The critical rolling displacement is often expressed as the critical rolling angle $\theta_{\text {crit }}=\xi_{\text {crit }} / R$, shown experimentally by [29] to be between $19-64 \times 10^{-3}$ rad for micron-scale polymer spheres. The rolling resistance model by Dominik and Tielens was also shown to produce good approximations of tangential force.

\section{RESULTS}

To validate different aspects of the normal and tangential collision models, the predictions of the DEM code are compared with separate sets of experimental data. To validate the normal adhesive and plastic deformation predictions of the TN model, the DEM is compared with the rebound of adhesive microspheres undergoing entirely normal collisions in the following subsection. As shown by [10], rebound trajectory is highly dependent on particle sphericity. Therefore, experimental data of particles that are known to be highly non-spherical, e.g. Arizona Road Dust (ARD) and quartz from [19] and [30], are not used for validation here as for other bounce stick models, e.g. [6]. It is noted that the effect of tangential deformation on normal adhesive effects, described theoretically by [31], cannot be validated by the normal collision data considered here. Once the model is extended to include particle non-sphericity, validation can be performed against existing micron-scale oblique collision data and the accuracy of the DEM approach in this context determined. The same result could be achieved by through experimental investigations of oblique, micron-scale spherical impacts. A method to account for this effect is presented in [26], which would not require significant changes to the DEM approach described here.

The tangential model and plastic deformation predictions of the TN model are validated using collision data from the oblique impacts of millimetre-scale spheres to ensure sphericity, with rolling adhesion considered separately given the reduced dependency of rebound on adhesive effects for diameters $\gg 10 \mu \mathrm{m}$ [11]. When considering data from millimetre-scale particles it should be noted that [6] showed that normal coefficient of restitution has no dependency on particle diameter when neglecting adhesion. The predictions of the DEM are not compared with those from existing bounce-stick models as, with varying dependencies on parametric fitting, it would be difficult for reasonable comparisons to be made. Arguably, approaches like those described in $[6,7,11]$ are most applicable for use in industry with reference to experimental data. Hence, predictions where input data is predominantly derived from published sources would not allow comparisons with the present model that represent their accuracy in industrial simulations. Similarly, maximum fitting of all models to available data would not present an opportunity to assess the accuracy of their physical modelling. Therefore, the focus of this paper is to assess the accuracy of the DEM approach with reference to specific physical phenomena.

\section{Normal Collisions of Microspheres}

The results of the DEM bounce stick model are compared with the experimental data from [12] to validate the modelling of adhesive effects. The TN model was compared with this data in [26] and [32], which required a dynamic yield stress to match 
experimental data (a similar technique to [6] and [11]). In [26], the yield stress varied from approximately $40 \mathrm{MPa}$ at a normal impact velocity of $1 \mathrm{~ms}^{-1}$ to approximately $145 \mathrm{MPa}$ at $30 \mathrm{~ms}^{-1}$ and above. In [11], Whitaker and Bons present a compelling argument for strain rate effects affecting the yield stress used in modelling. As they explain, for some materials the onset of plastic deformation is known to be dependent on the rate at which the material is deformed [33]. However, the variation modelled in [26] seems extreme. Neither the TN model nor [11] explicitly include viscoelasticity, but rather match physical phenomena related to strain rate by varying the yield stress. In this paper the impact of different viscoelastic dissipation coefficients $\eta_{\mathrm{n}}$ is studied to provide a more robust explanation of the experimental dependence on strain rate. The effects of squeeze-film damping are also considered with two values of minimum separation gap $\delta_{\text {gap }}$ considered, which is analogous in this context to surface roughness height.

In [12], ammonium fluorescein spheres, herein referred to as $\mathrm{NH} 4$, between $2.58 \mu \mathrm{m}$ and $6.88 \mu \mathrm{m}$ in diameter were accelerated towards a flat plate at a normal angle of incidence. Total velocity varied between $1-100 \mathrm{~ms}^{-1}$. Different substrate materials were used, with silicon and molybdenum considered here. Material properties for the particles and substrates are shown in Table 1 . Note that $\gamma$ is only shown for substrates as it depends on the particle-substrate material pairs, density is only shown for the particle as substrate mass is not relevant and it is assumed that, for these material pairs, the particle will always deform before the substrate hence only the particle yield stress is shown. The minimum separation gap $\delta_{\text {gap }}$ was set to $1 \times 10^{-9} \mathrm{~m}$ for the results shown in Figures 4 - 7, a similar value to that used in [14] for the same experimental data. Additional simulations were also run for $\delta_{\text {gap }}=1 \times 10^{-10} \mathrm{~m}$ which, although unfeasibly smooth in reality, demonstrates the upper limit of the squeeze-film effect through the dependence on the ratio $\delta_{\text {gap }} / x_{0}$ in Equations 21 and 22. The two squeeze-film conditions are therefore referred to as $\mathrm{SF}=0.1$ and $\mathrm{SF}=0.01$ respectively where SF is the ratio $\delta_{\text {gap }} / x_{0}$. The dynamic viscosity of the air surrounding the particle was set to $1.5 \times 10^{-5} \mathrm{Pas}$ as in [14].

As described in the Methodology section, the normal viscoelastic dissipation coefficient $\eta_{\mathrm{n}}$ is calculated using $e_{0}$, equivalent to the $\mathrm{CoR}_{\mathrm{n}}$ if viscoelasticity were the only dissipation mechanism in a normal contact. DEM predictions of $\mathrm{CoR}_{\mathrm{n}}$ were generated for $4.90 \mu \mathrm{m} \mathrm{NH} 4$ spheres impacting a molybdenum substrate with different values of $e_{0}$, shown in Figure 4. Simulations ran for approximately 25,000 iterations with a runtime of approximately $4 \mathrm{~s}$ per impact. $\mathrm{CoR}_{\mathrm{n}}$ is shown to have a significant dependence on viscoelasticity, through $e_{0}$, with $\mathrm{CoR}_{\mathrm{n}}$ varying between near unity and zero between $e_{0}=1.0$ and 0.10 . Notably, the TN model is able to match experimental data well with a constant yield stress when $e_{0}=0.78$. In addition to direct viscoelastic

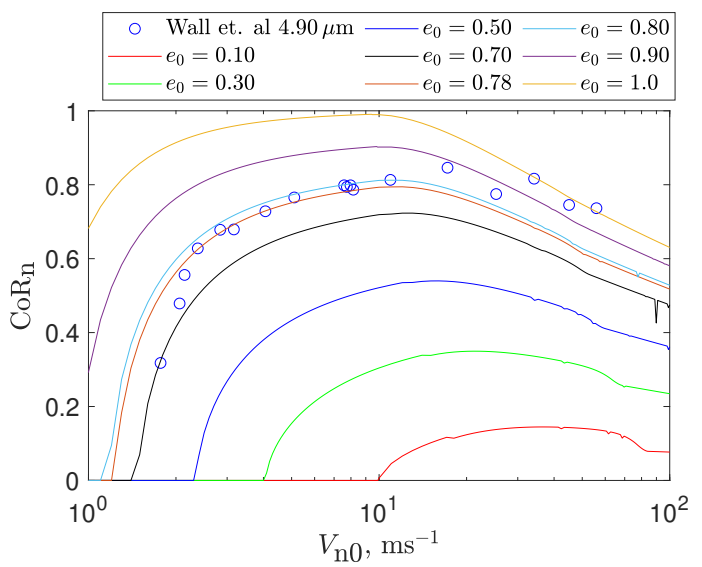

FIGURE 4. NORMAL COLLISIONS OF $4.90 \mu \mathrm{m}$ NH4 SPHERES AND A MOLYBDENUM SUBSTRATE FOR VARYING VISCOELASTICITY, EXPERIMENTAL DATA FROM [12].

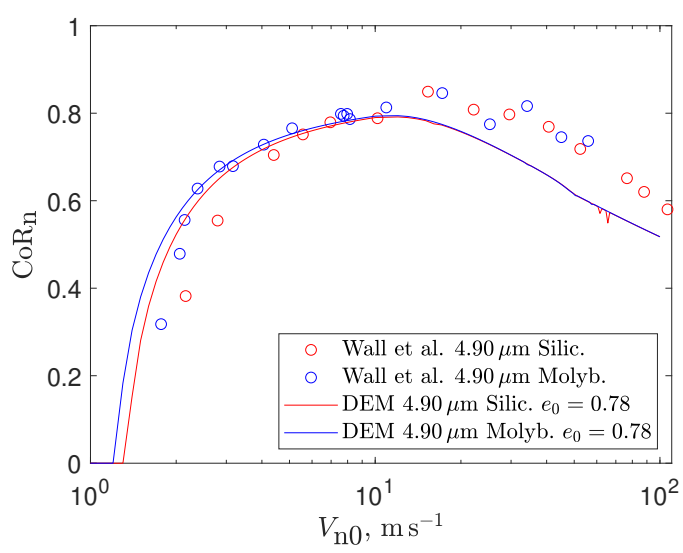

FIGURE 5. NORMAL COLLISIONS OF $4.90 \mu \mathrm{m}$ NH4 SPHERES [12] WITH MOLYBDENUM AND SILICON SUBSTRATES.

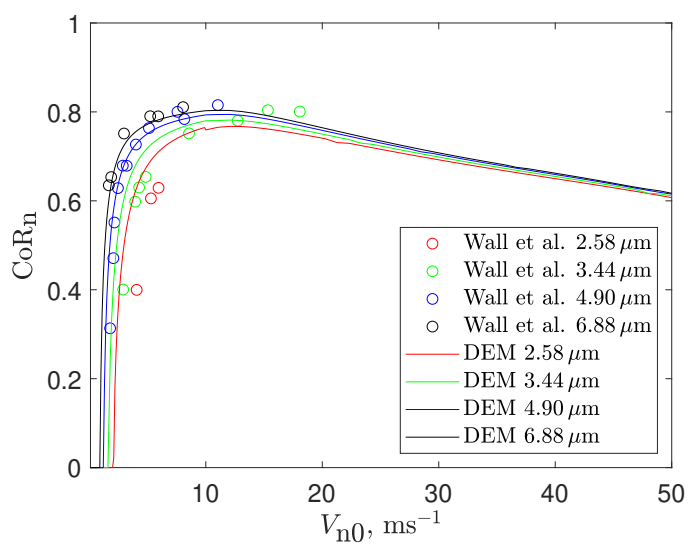

FIGURE 6. NORMAL COLLISIONS OF NH4 MICROSPHERES WITH A MOLYBDENUM SUBSTRATE, $e_{0}=0.78, \mathrm{SF}=0.1$. EXPERIMENTAL DATA FROM [12]. 
TABLE 1. NH4 PARTICLE AND SUBSTRATE MATERIALS PROPERTIES FROM [12] AND [32].

\begin{tabular}{lccc}
\hline & NH4 & Molybdenum & Silicon \\
\hline$E(\mathrm{GPa})$ & 1.2 & 330 & 182 \\
Poisson's Ratio & 0.33 & 0.38 & 0.28 \\
Density $\left(\mathrm{kg} \mathrm{m}^{-3}\right)$ & 1350 & - & - \\
$\gamma\left(\mathrm{J} \mathrm{m}^{-2}\right)$ & - & 0.34 & 0.38 \\
$\sigma_{\mathrm{y}}(\mathrm{MPa})$ & 74 & - & - \\
\hline
\end{tabular}

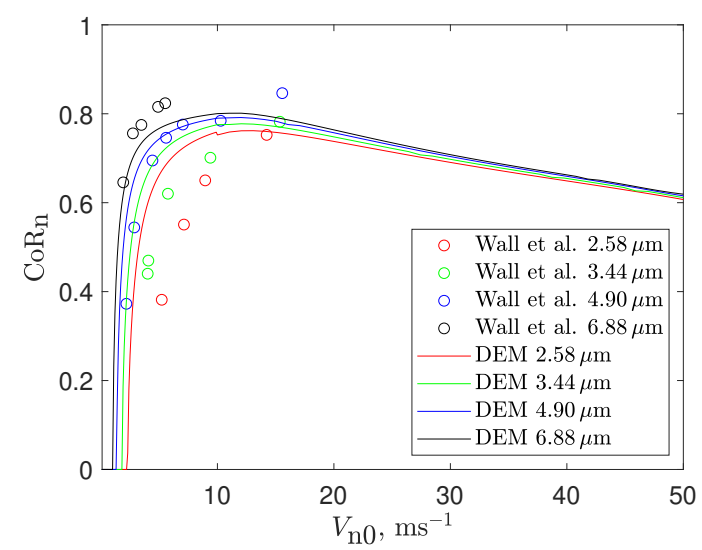

FIGURE 7. NORMAL COLLISIONS OF NH4 MICROSPHERES WITH A SILICON SUBSTRATE, $e_{0}=0.78, \mathrm{SF}=0.1$. EXPERIMENTAL DATA FROM [12].

dissipation, an additional viscoelastic phenomenon leads to adhesive energy dissipation. During an elastic collision, the particle behaves as a spring storing elastic potential energy. Therefore, at the point where first contact is made, the point of zero normal overlap, the particle should have approximately the same magnitude of normal velocity on rebound and incidence, assuming small losses due to direct viscoelastic dissipation. However, with adhesive contact the particle remains adhered to the surface past zero normal overlap during rebound, "necking" as kinetic energy is transferred back to elastic potential energy. It is this elastic spring that provides the necessary potential energy to overcome the adhesive potential and allow rebound. Hence, the adhesive process is heavily dependent on particle deformation at a point of high normal velocities, noting that the normal dissipative force $F_{\mathrm{nd}}$ is the product of $\eta_{\mathrm{n}}$ and $V_{\mathrm{n}}$.

DEM predictions of $\mathrm{CoR}_{\mathrm{n}}$ for a silicon substrate are also shown in Figure 5, where $e_{0}=0.78$ was found to produce the best fit to experimental data. This fit was obtained using only the mechanical properties provided by $[12,32]$, without further fitting of $\sigma_{\mathrm{y}}$ to the results. The DEM correctly predicts a higher sticking velocity of the NH4-silicon pair, given the higher surface free energy between the two. The coefficients of restitution converge when in the plastic impaction regime, as is also the case for the experimental data. Further simulations are shown in Figures 6 and 7 , where the particle diameter ranges from $2.58-6.88 \mu \mathrm{m}$ for molybdenum and silicon substrates respectively. The critical sticking velocity increases with particle diameter, matching the results of existing bounce stick models $[6,7]$. The value of $e_{0}$ was calibrated for $4.90 \mu \mathrm{m}$ particles, so while $\operatorname{CoR}_{n}$ matches these data extremely well the DEM tends to over-predict $\mathrm{CoR}_{\mathrm{n}}$ for smaller particles and under-predict $\mathrm{CoR}_{\mathrm{n}}$ for larger diameters. At $\mathrm{SF}=0.1$ squeeze-film damping clearly arrests the motion of small particles disproportionately, due to the dependence on collision Stokes number, Equation 20, providing a possible explanation for the discrepancy between modelling and experimental data. To understand the sensitivity to SF, SF was reduced by an order of magnitude to 0.01: the ratio of $\mathrm{CoR}_{n}$ is shown in Figure 8. While the normal coefficient of restitution is largely unchanged (ratio $\approx 1$ ), the magnitude of the effect is greater as particle diameter decreases and as the normal velocity approaches the stick velocity $\mathrm{CoR}_{\mathrm{n}}=0$ for all particles. Indeed, excluding the squeeze-film effects entirely from Figure 6 does not significantly improve the fit to data, especially if the value of $e_{0}$ is adjusted to compensate. It is also noteworthy that these particles are relatively smooth and spherical, meaning the effects of squeeze-film damping are likely stronger here than for the rough, non-spherical particles encountered in engines.

The DEM predictions of $\mathrm{CoR}_{n}$ match the key features of experimental results for a range of particle diameters and collision velocities considering adhesive, viscoelastic and inelastic effects. Squeeze-film damping is seen to have only a small effect on the normal coefficient of restitution. It is therefore recognised that the model fails to capture another diameter-related phenomenon which affects particle motion. While it might be possible to use both a velocity dependent yield stress and variable viscoelastic coefficient to perfectly replicate particle rebound, suitable physical bases for doing so are not yet apparent. As a key motivation for the development of the model is to allow its application to different particles without empirical tuning this has not been undertaken.

Comparison to Existing Models Predictions of $\mathrm{CoR}_{\mathrm{n}}$ from DEM were compared with those from The Ohio State University (OSU) model [6] and the Critical Velocity (CV) model [7]. As explained previously, these models are primarily energy based and require some degree of empirical tuning using 


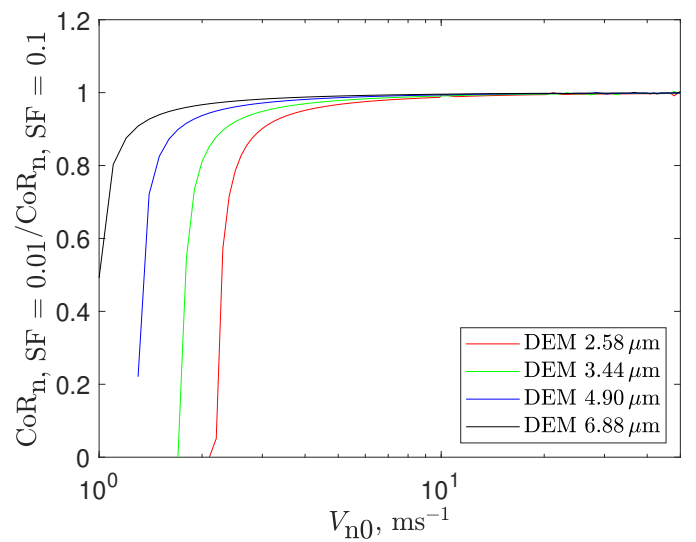

FIGURE 8. EFFECT OF DIFFERENT LEVELS OF SQUEEZEFILM DAMPING ON NORMAL COEFFICIENT OF RESTITUTION FOR THE COLLISIONS SHOWN IN FIGURE 6.

bespoke material properties. When comparing the predictions of largely empirical models and those of the predominantly deterministic DEM method, it is important to consider how these approaches may be used in industrial simulations of particle deposition: extensive collision rebound data are unlikely to be available, especially for novel material pairs. Hence, models should prioritise the use of published materials data and minimise empirical corrections. Predictions from the energy-based methods presented here are, therefore, generated using optimised input parameters that are constant over the full range of collision velocities modelled. This allows the fidelity of the models' physics to be assessed independently of the quality of available material properties. The same procedure has been used to the optimise $e_{0}$ in the DEM model and thus provides a fair comparison between methods.

Two primary parameters that influence $\operatorname{CoR}_{\mathrm{n}}$ are $\gamma$ and $\sigma_{\mathrm{y}}$, which define both loss mechanisms that affect normal rebound velocity in the OSU model, plastic deformation and adhesion. These were optimised against $4.90 \mu \mathrm{m} \mathrm{NH} 4$ spheres vs. a silicon substrate from [12] as this presented the largest range of collision velocities available. Yield stress is also explicitly tuned in $[6,11]$ while $\gamma$ is not well reported in literature. The optimised values were defined as those which generated a minimum average error between model predictions and experimental data in the range $0.1 \mathrm{MPa} \leq \sigma_{\mathrm{y}} \leq 100 \mathrm{MPa}$ and $0.01 \mathrm{Jm}^{-2} \leq \gamma \leq 1 \mathrm{Jm}^{-2}$. One thousand samples were taken of both $\gamma$ and $\sigma_{\mathrm{y}}$ to produce a $10^{6}$ elements map of $\mathrm{CoR}_{\mathrm{n}}$. A global least squares minimisation yielded $\sigma_{\mathrm{y}}=67.2 \mathrm{MPa}$ and $\gamma=0.0536 \mathrm{Jm}^{-2}$. The same procedure was followed for the $\mathrm{CV}$ model, where the parameters optimised were $\gamma=0.328 \mathrm{Jm}^{-2}$ and $e_{0}=0.848$. The notable discrepancy in $\gamma$ between the OSU model and other approaches arises from a difference in calculations of assumed maximum

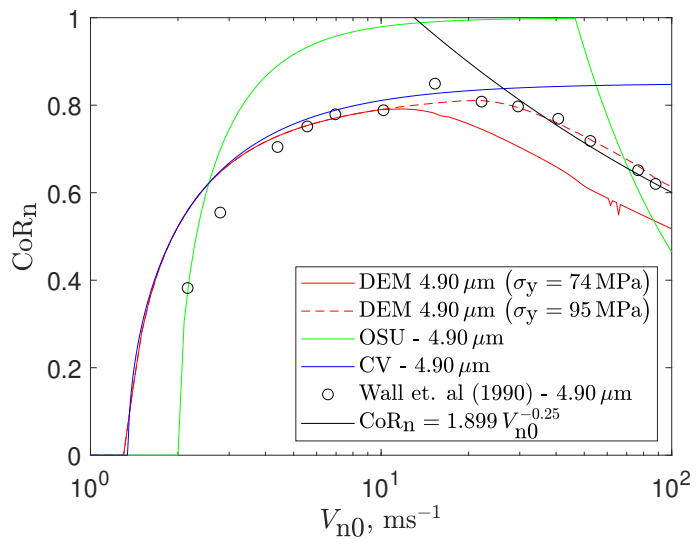

FIGURE 9. COMPARISON BETWEEN THE DEM, OSU [6] AND CRITICAL VELOCITY (CV) [7] DEPOSITION MODELS FOR $4.90 \mu \mathrm{m}$ NH4 PARTICLES VS. SILICON.

contact area, the OSU model taking an empirical approach that predicts larger values than Hertzian methods when using the constants $a, b$, and $c$ supplied by [6].

The predictions of $\mathrm{CoR}_{\mathrm{n}}$ for $4.90 \mu \mathrm{m}$ spheres using each model with the optimised parameters are shown in Figure 9. Here the value of $\sigma_{\mathrm{y}}$ used in the DEM model was optimised to $95 \mathrm{MPa}$. The performance of the CV and DEM models at low velocities are very similar, owing to their shared use of Hertzian contact models. The CV model's use of an empirical $\mathrm{CoR}_{n}$ in the absence of adhesion, $e_{0}$, approximates viscoelastic behaviour, though it is not physically modelled as in the DEM, instead being employed as an empirical correction. As plastic deformation is neglected, the $\mathrm{CV}$ model fails to accurately predict the drop in $\mathrm{CoR}_{\mathrm{n}}$ at high velocities. By contrast, the OSU model predicts plastic deformation but the inability to account for strain-rate effects when a constant value of $\sigma_{\mathrm{y}}$ is used reduces accuracy at intermediate collision velocities. Analytical solutions of collision energetics, $[24,34]$ show that $\operatorname{CoR}_{\mathrm{n}}$ is proportional to $V_{\mathrm{n} 0}^{-0.25}$ during highly plastic deformation, confirmed by several experimental studies summarised in [26]. This trend is plotted in Figure 9, fitted to the experimental data point at the highest velocity $V_{\mathrm{n} 0} \approx 90 \mathrm{~ms}^{-1}$. The plastic behaviour is close to DEM predictions, however the gradient of the OSU CoR ${ }_{n}$ is significantly steeper than the analytical relationship. Using a constant $\sigma_{\mathrm{y}}$ and $\gamma$, there does not appear to be a close fit of the OSU model to experimental data however this was accounted for using a velocity dependent $\sigma_{\mathrm{y}}$ fit in [11]. Overall, the performance of DEM is shown to be favourable compared to existing models where optimised values are held constant over a range of collision velocities. 
TABLE 2. PARTICLE-SUBSTRATE MATERIALS PROPERTIES.

\begin{tabular}{lllll}
\hline & Alumina & $\begin{array}{l}\text { Float } \\
\text { Glass }\end{array}$ & Al7178 & $\begin{array}{l}\text { Hard. } \\
\text { Steel }\end{array}$ \\
\hline$E(\mathrm{GPa})$ & 360 & 70.0 & 73.1 & - \\
$G(\mathrm{GPa})$ & - & - & - & 80.0 \\
Poisson's Ratio & 0.23 & 0.25 & 0.33 & 0.29 \\
Density $\left(\mathrm{kg} \mathrm{m}^{-3}\right)$ & $3500^{*}$ & - & - & - \\
$\sigma_{\mathrm{y}}(\mathrm{MPa})$ & - & 1700 & 470 & 1320 \\
$\mu$ & - & 0.0920 & 0.180 & 0.163 \\
References & {$[35]$} & {$[35,37]$} & {$[35,38]$} & {$[36,39]$} \\
\hline
\end{tabular}

${ }^{*}$ porosity $=0.68$ for hardened steel cases

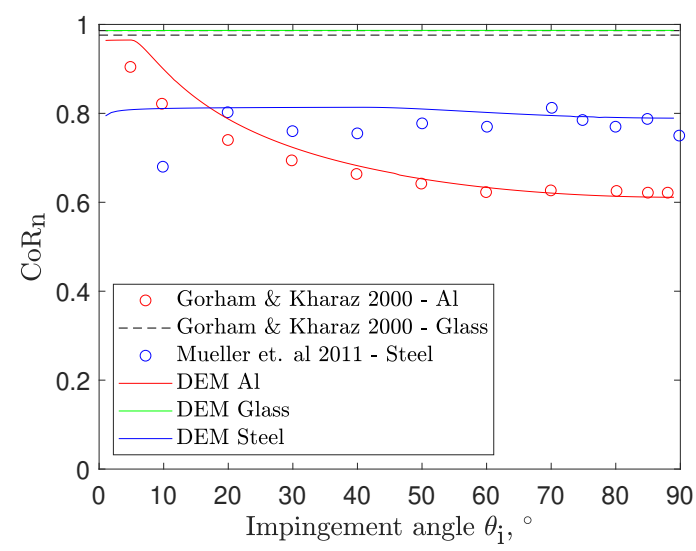

FIGURE 10. NORMAL COEFFICIENTS OF RESTITUTION OF MILLIMETRE-SCALE PARTICLES, EXPERIMENTAL DATA FROM $[35,36]$. DASHED LINES SHOW EXPERIMENTAL RANGE.

\section{Millimetre-Scale Sphere Collisions}

The collisions of millimetre-scale particles were simulated to validate the tangential model [17] using experimental data from $[35,36]$. Large alumina particles $\left(5.0 \mathrm{~mm}\left(V_{0}=3.9 \mathrm{~ms}^{-1}\right)\right.$ and $1.8 \mathrm{~mm}\left(V_{0}=2.3 \mathrm{~ms}^{-1}\right)$ ensure sphericity and allow comparison between experimental and numerical angular velocities at rebound, as experimental techniques prohibit measurements for micron-scale particles. As the particles are large, squeezefilm effects are likely negligible and hence neglected. Particle and substrate materials properties are shown in Table 2. All simulations were run with $\gamma=0.3 \mathrm{Jm}^{-2}$ as the value is likely to be of order unity, although adhesion is likely small at this scale.

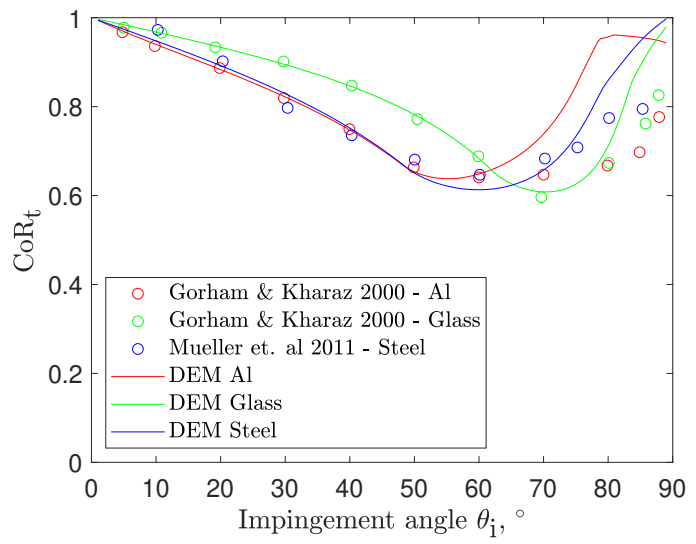

FIGURE 11. TANGENTIAL COEFFICIENTS OF RESTITUTION OF MILLIMETRE-SCALE PARTICLES, EXPERIMENTAL DATA FROM [35] AND [36].

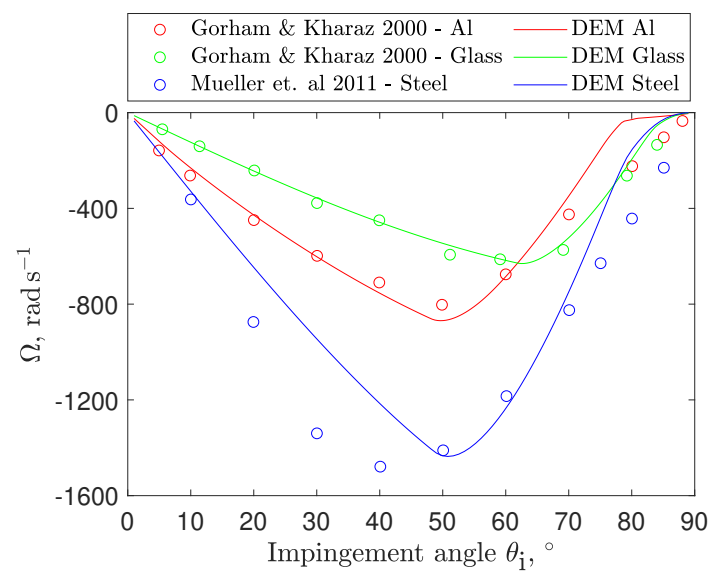

FIGURE 12. REBOUND ANGULAR VELOCITY OF MILLIMETRE-SCALE PARTICLES, EXPERIMENTAL DATA FROM [35] AND [36].

The rebound properties of the millimetre scale particles are shown in Figures 10-12. The DEM predictions of normal coefficient of restitution in Figure 10 show good agreement with experimental data. Increasing plastic deformation with more normal collisions is modelled well in the aluminium substrate case, while the DEM correctly predicts elastic collisions with a glass substrate, the $\mathrm{CoR}_{\mathrm{n}}$ being close to unity. Rebound in the alumina-steel case is largely driven by viscoelasticity where $e_{0}=0.75$, with negligible plastic deformation demonstrated by the lack of dependency on normal velocity at high impingement angles. This is consistent with the description of the aluminasteel collisions in [36] as "elastic-dominant". Values of $e_{0}$ for the aluminium and glass cases were 0.95 and 0.98 respectively, within the range of $0.90<\mathrm{CoR}_{n}<1.0$ shown by [40] to be 
responsible for reductions in $\mathrm{CoR}_{\mathrm{n}}$ related to viscoelastic losses and elastic wave propagation. While $e_{0}$ for the alumina-steel collisions is much lower than that of the alumina-aluminium and alumina-glass impacts, the near constant $\mathrm{CoR}_{n}$ with impingement angle and apparent lack of plastic deformation makes it likely that only viscoelasticity is responsible, given the size of the particles precludes adhesive effects. It is possible that material fracture or particle non-sphericity are also responsible, as [36] notes the particles have a sphericity of only 0.95 . Indeed, [10] demonstrate that non-sphericity reduces average $\mathrm{CoR}_{\mathrm{n}}$ across a range of particle orientations, however this is excluded from the present analysis. The value of $\sigma_{\mathrm{y}}$ for glass in Table 2 was taken as $10 \%$ of the maximum theoretical value presented in [37], as the author acknowledges that small surface areas will have higher strengths than typical values used in design.

Predictions of $\mathrm{CoR}_{\mathrm{t}}$ in Figure 11 show excellent agreement at oblique impact angles, where the particle slides throughout the duration of the impact, however the DEM diverges from experimental data at impact angles close to normal. Excellent agreement is shown in the elastic glass and steel substrate cases, while comparison with $\mathrm{CoR}_{\mathrm{n}}$ in Figure 10 indicates that increasing plastic deformation reduces the ability of Cundall and Strack's model to accurately model $\mathrm{CoR}_{\mathrm{t}}$. As described by [41], when using normal force models that consider plastic deformation the tangential spring in Equation 24 may not always be constrained by Coulomb friction $\left(\mu F_{n}\right)$. Therefore, adaption of Cundall and Strack's model will be considered in further work to improve $\mathrm{CoR}_{\mathrm{t}}$ accuracy at near-normal angles of incidence. Angular velocity is well predicted by the DEM, as shown by Figure 12. Agreement is best where substrate and particle material properties are provided with experimental rebound data, as is the case for the aluminium and glass collision. As described by $[9,42]$, the location of peak angular velocity in Figure 12 is largely governed by elastic materials properties. Where these are not provided with experimental data and hence must be found elsewhere, e.g. the steel substrate case, less accurate predictions can be made.

Overall, the DEM is shown to accurately predict $\mathrm{CoR}_{\mathrm{n}}, \mathrm{CoR}_{\mathrm{t}}$ and $\Omega$ for different materials pairs considering viscoelasticty, friction and plastic deformation. Data matching, conducted to estimate $\eta_{n}$ at a single particle size appears to scale robustly throughout the data. The model is highly sensitive to material properties and future extensions should address the applicability of Cundall and Strack's tangential model to better predict $\operatorname{CoR}_{\mathrm{t}}$ at the most normal impingement angles where plastic deformation is significant.

Comparison to Existing Models The OSU model adopts the impulse-based tangential modelling strategy from the CV ap-

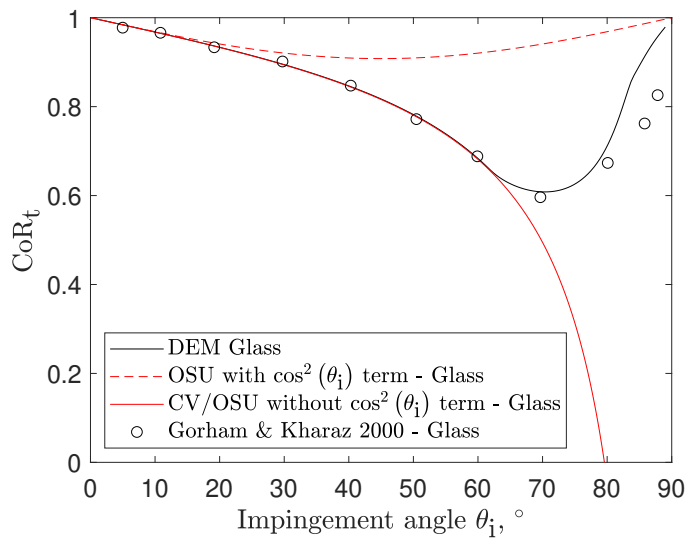

FIGURE 13. PREDICTED $\mathrm{CoR}_{\mathrm{t}}$ FOR DEM VS. OSU [6] AND CRITICAL VELOCITY [7]. EXPERIMENTAL DATA FROM [35].

proach [7]. At oblique impingement angles where the particle slides throughout the contact duration, this is an analytical solution. DEM predictions of tangential restitution were compared to those of the OSU model, with and without the empirical $\cos ^{2}\left(\theta_{\mathrm{i}}\right)$ correction proposed by the authors. Angular velocity was not compared as this was not modelled by $[6,11]$. Model predictions were generated for the alumina-glass collision case shown above, from [35]. Material properties were not optimised as in the previous subsection because the experimental data has no significant dependence on $\sigma_{\mathrm{y}}$ or $\gamma$. Results are shown in Figure 13, where both modelling approaches accurately predict $\operatorname{CoR}_{t}$ at low impingement angles where the particle slides throughout the duration of contact. However, the simple impulsive approach applied by the OSU and CV models is unable to capture the transition from sliding to rolling or the tangential compliance of the contact patch at higher impingement angles [41]. The empirical $\cos ^{2}\left(\theta_{\mathrm{i}}\right)$ term reduces overall model accuracy and does not appear to represent the underlying collision physics. The DEM model performs favourably when compared to existing models, especially at high impingement angles.

\section{Rolling Adhesion}

Rolling adhesion reduces the magnitude of the particle tangential velocity by reducing the angular velocity, as shown in Equations 3 and 26. Due to the difficulties in measuring the angular velocity of micron-scale particles, it is not possible to directly compare DEM predictions of rolling adhesion with experimental data. However, as previously discussed, [29] found that the rolling adhesion model of Dominik and Tielens [28] showed good agreement with experimental measurements where micron-scale particles were rolled along a surface with a stylus. 
Here, the effect of rolling adhesion is shown for a $10 \mu \mathrm{m} \mathrm{di}-$ ameter particle of ARD using materials properties from [6] at $\theta_{\mathrm{i}}=45^{\circ}$ and $\gamma=0.8$. The critical value of rolling displacement $\xi=\xi_{\text {crit }}$ is set to $0.06 R$ to match the experimental observations of micron-scale particles by [29]. A comparison between the rolling and tangential velocites of the particle while sticking is shown in Figure 14. The particle initially slides along the surface until the tangential and rolling velocities are matched, at which point the contact patch enters microslip at approximately $t=0.3 \times 10^{-7} \mathrm{~s}$. In this regime, the contact patch oscillates akin to a spring-dashpot system as described by Cundall and Strack with the oscillations damped by friction and tangential viscoelasticity, leading the particle to roll according to rigid body motion. As the slip velocity between the particle and wall reduces, the rolling adhesion moment $M_{\mathrm{r}}$, Equation 26, becomes the dominant source of tangential friction and hence is responsible for the particle coming to rest. This is demonstrated by the near constant tangential velocity of the particle when rolling once rolling friction is disabled, also in Figure 14. However, the distance a stuck particle rolls after impact is not relevant to broader bounce stick modelling, only the effect of rolling adhesion on $\mathrm{CoR}_{t}$ is important. The effects of rolling adhesion on tangential velocity will increase with collision time, given $\xi$ is the time integral of the rolling velocity. As, through impulse, the largest collision time will be at the lowest possible normal velocity at which rebound still occurs, simulations were performed at $\theta_{\mathrm{i}}=45^{\circ}$ just above the critical velocity of $2.4 \mathrm{~m} \mathrm{~s}^{-1}$ with and without rolling adhesion enabled. The results are shown in Figure 15 where the collision time is much less than that taken for the particle to come to rest in Figure 14. Rolling adhesion has only a small effect on tangential velocity at rebound and hence $\operatorname{CoR}_{\mathrm{t}}$ is $1.2 \%$ lower than with rolling adhesion at 0.711 vs. 0.719 . It should also be noted that $\xi$ reached a maximum value of only $0.14 \xi_{\text {crit }}$, reducing the sensitivity of the DEM approach to experimental observations from [29]. Therefore, while it is not possible to quantitatively validate the modelling of rolling adhesion, the implementation here shows the correct qualitative behaviour using a model that has been shown to match experimental data for adhesive rolling moments. In addition, in the limiting case applicable to gas turbine particulates the effect on $\mathrm{CoR}_{\mathrm{t}}$ was found to be small.

\section{CONCLUSIONS}

A model has been presented that can predict the rebound trajectory and angular velocity of spherical particles considering inelastic, viscoelastic and adhesive behaviour. These represent all relevant first-order physical phenomena for the rebound of spherical particulates in gas turbine secondary air systems. Predictions were accurate when compared to experimental data across a range of particle diameters, velocities and impingement angles applicable to gas turbine components. The model is largely deterministic, directly simulating the relevant collision

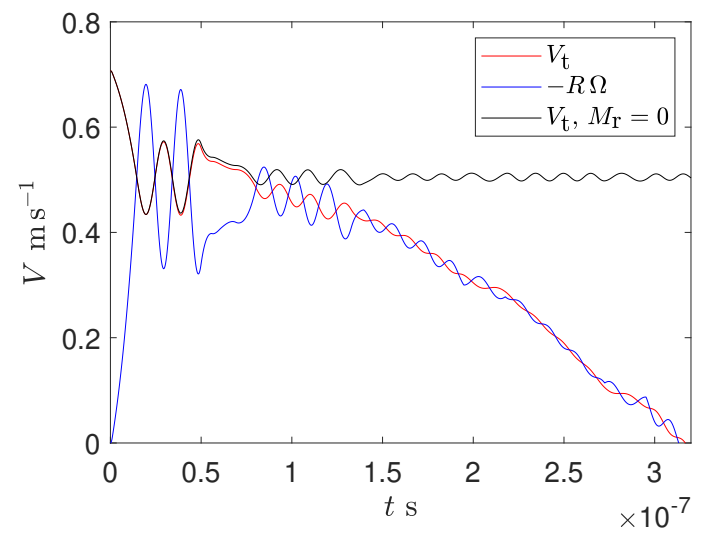

FIGURE 14. TANGENTIAL AND ROLLING VELOCITIES OF A $10 \mu \mathrm{m}$ ARD PARTICLE DURING A STICKING COLLISION.

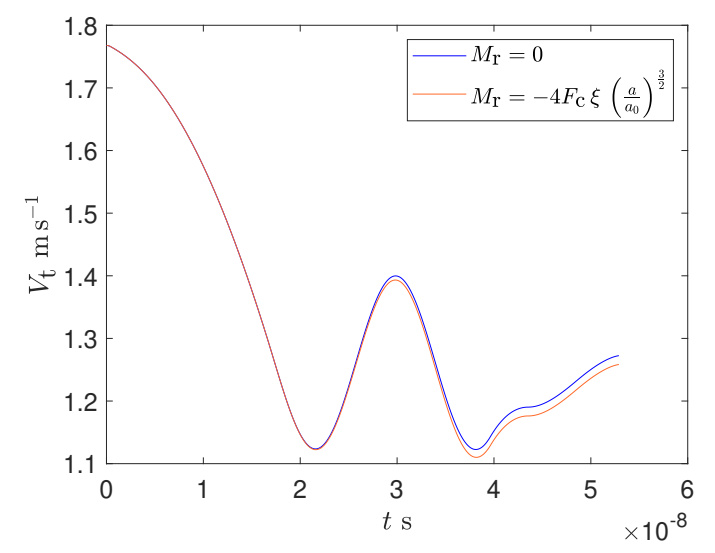

FIGURE 15. TANGENTIAL VELOCITIES OF A $10 \mu \mathrm{m}$ ARD PARTICLE DURING BOUNCE COLLISIONS WITH AND WITHOUT ROLLING ADHESION ENABLED AT $\theta_{\mathrm{i}}=45^{\circ}$.

physics that occur throughout the contact time and hence can be used with almost entirely with published materials properties. External effects like temperature and surface roughness can be included through augmented input parameters, while the effects of squeeze-film damping and rolling adhesion were found to be negligible for cases applicable to gas turbines.

Previous work has shown the importance of collision-induced rotation and coefficient of restitution on rebound trajectory and deposition. The first inclusion and validation of these parameters in a bounce stick model will allow far superior predictions of particle transport where many repeated collisions occur, as is likely the case in gas turbine components. Model predictions compare favourably with existing energy-based methods which fail to capture the underlying collision physics. The inclusion 
of viscoelastic dissipation in particular is shown to remove a dependency of yield stress on non-physical parameters. However, fitting the viscoelastic dissipation coefficient proposed to experimental results suggests that this may itself integrate a number of physical phenomena and further work is required to reduce dependency on empirical data. The force-based, explicit nature of the DEM presents a flexible and adaptable platform for the inclusion of further physical phenomena like the modelling of arbitrarily-shaped particles, the physics of which are highly-coupled and have been shown in previous work to differ from that of spheres.

While the DEM approach as described here could be implemented directly into CFD, the computational expense means application through a machine learning surrogate model is preferred for simulations of the complexity expected by industry. Preliminary implementations of this approach show promising results and will be presented in further work that describes the full workflow of incorporating this implementation of DEM into commercial solvers. Without a suitable particle fragmentation or erosion model, the approach as described here is most applicable to modelling deposition of small particles at high temperatures where deformation remains ductile. Therefore it is most readily applied to the secondary air and cooling systems downstream of the compressor. However, due to the differing collision physics of spherical vs. non-spherical particles, it is recommended that particle non-sphericity is included in bounce-stick models before application to particulate flows in gas turbine components.

\section{ACKNOWLEDGMENT}

The authors gratefully acknowledge the financial support and collaboration of Rolls-Royce Plc and the ATI through the MALIT programme, and the funding of the lead author through the EPSRC and the Centre for Doctoral Training in Gas Turbine Aerodynamics.

\section{REFERENCES}

[1] Dunn, M. G., 2012. "Operation of gas turbine engines in an environment contaminated with volcanic ash". Journal of Turbomachinery, 134(5), p. 051001.

[2] Vadgama, N., 2021. "On atmospheric particulate deposition in gas turbines (thesis submitted)". PhD thesis.

[3] Chou, C., Formenti, P., Maille, M., Ausset, P., Helas, G., Harrison, M., and Osborne, S., 2008. "Size distribution, shape, and composition of mineral dust aerosols collected during the african monsoon multidisciplinary analysis special observation period 0: Dust and biomass-burning experiment field campaign in Niger, January 2006". Journal of Geophysical Research, 113.

[4] Reid, E. A., 2003. "Characterization of African dust transported to Puerto Rico by individual particle and size seg- regated bulk analysis". Journal of Geophysical Research, 108(D19).

[5] Clarkson, R. J., Majewicz, E. J., and Mack, P., 2016. "A reevaluation of the 2010 quantitative understanding of the effects volcanic ash has on gas turbine engines". Proceedings of the Institution of Mechanical Engineers, Part G: Journal of Aerospace Engineering, 230(12), p. 2274-2291.

[6] Bons, J. P., Prenter, R., and Whitaker, S., 2017. "A simple physics-based model for particle rebound and deposition in turbomachinery". Journal of Turbomachinery, 139(8), p. 081009.

[7] Brach, R. M., and Dunn, P. F., 1992. "A mathematical model of the impact and adhesion of microspheres". Aerosol Science and Technology, 16(1), p. 51-64.

[8] Yu, K., and Tafti, D., 2019. "Size- and temperaturedependent collision and deposition model for micron-sized sand particles". Journal of Turbomachinery, 141(3), p. 031001.

[9] Gaskell, J. G., Vadgama, N., McGilvray, M., Gillespie, D. R. H., and Clarkson, R. J., 2020. "Collision-induced rotation and the Magnus force on particulates". AIAA Aviation 2020 Forum.

[10] Gaskell, J. G., McGilvray, M., and Gillespie, D. R. H., 2021. "The rebound of non-spherical particles in gas turbine components. Proceedings of the $14^{\text {th }}$ European Turbomachinery Conference (manuscript accepted)".

[11] Whitaker, S. M., and Bons, J. P., 2018. "An improved particle impact model by accounting for rate of strain and stochastic rebound". Volume 2D: Turbomachinery.

[12] Wall, S., John, W., Wang, H.-C., and Goren, S. L., 1990. "Measurements of kinetic energy loss for particles impacting surfaces". Aerosol Science and Technology, 12(4), p. 926-946.

[13] Marshall, J. S., 2011. "Viscous damping force during headon collision of two spherical particles". Physics of Fluids, 23(1), p. 013305.

[14] Marshall, J. S., 2018. "Modeling and sensitivity analysis of particle impact with a wall with integrated damping mechanisms". Powder Technology, 339, p. 17-24.

[15] Joseph, G. G., Zenit, R., Hunt, M. L., and Rosenwinkel, A. M., 2001. "Particle-wall collisions in a viscous fluid". Journal of Fluid Mechanics, 433, p. 329-346.

[16] Cundall, P. A., 1971. "The measurement and analysis of accelerations in rock slopes". PhD thesis.

[17] Cundall, P. A., and Strack, O. D. L., 1980. "Discussion: A discrete numerical model for granular assemblies". Géotechnique, 30(3), p. 331-336.

[18] Singh, S., and Tafti, D., 2015. "Particle deposition model for particulate flows at high temperatures in gas turbine components". International Journal of Heat and Fluid Flow, 52, p. 72-83. 
[19] Reagle, C. J., Delimont, J. M., Ng, W. F., and Ekkad, S. V., 2013. "Study of microparticle rebound characteristics under high temperature conditions". Journal of Engineering for Gas Turbines and Power, 136(1), p. 011501.

[20] Wammack, J. E., Crosby, J., Fletcher, D., Bons, J. P., and Fletcher, T. H., 2008. "Evolution of surface deposits on a high-pressure turbine blade-part I: Physical characteristics". Journal of Turbomachinery, 130(2).

[21] Sommerfeld, M., and Huber, N., 1999. "Experimental analysis and modelling of particle-wall collisions". International Journal of Multiphase Flow, 25(6-7), p. 1457-1489.

[22] Marshall, J., 2009. "Discrete-element modeling of particulate aerosol flows". Journal of Computational Physics, 228(5), p. 1541-1561.

[23] Thornton, C., and Ning, Z., 1998. "A theoretical model for the stick/bounce behaviour of adhesive, elastic-plastic spheres". Powder Technology, 99(2), p. 154-162.

[24] Johnson, K. L., 1985. Contact Mechanics. Cambridge University Press.

[25] Thornton, C., Cummins, S. J., and Cleary, P. W., 2013. “An investigation of the comparative behaviour of alternative contact force models during inelastic collisions". Powder Technology, 233, pp. $30-46$.

[26] Ning, Z., 1995. "Elasto-plastic impact of fine particles and fragmentation of small agglomerates". PhD thesis.

[27] Davis, R. H., Serayssol, J.-M., and Hinch, E. J., 1986. "The elastohydrodynamic collision of two spheres". Journal of Fluid Mechanics, 163, p. 479-497.

[28] Dominik, C., and Tielens, A. G. G. M., 1995. "Resistance to rolling in the adhesive contact of two elastic spheres". Philosophical Magazine A, 72(3), p. 783-803.

[29] Ding, W., Zhang, H., and Cetinkaya, C., 2008. "Rolling resistance moment-based adhesion characterization of microspheres". The Journal of Adhesion, 84(12), p. 996-1006.

[30] Bons, J. P., Blunt, R., and Whitaker, S., 2015. "A comparison of techniques for particle rebound measurement in gas turbine applications". Volume 1: Aircraft Engine; Fans and Blowers; Marine.

[31] Savkoor, A. K., and Briggs, G. A. D., 1977. "The effect of tangential force on the contact of elastic solids in adhesion".
Proceedings of the Royal Society of London. A. Mathematical and Physical Sciences, 356(1684), p. 103-114.

[32] Xie, J., Dong, M., Li, S., Shang, Y., and Fu, Z., 2017. "Dynamic characteristics for the normal impact process of micro-particles with a flat surface". Aerosol Science and Technology, 52(2), p. 222-233.

[33] Heard, H. C., 1963. "Effect of large changes in strain rate in the experimental deformation of yule marble". The Journal of Geology, 71(2), p. 162-195.

[34] Thornton, C., 1997. "Coefficient of restitution for collinear collisions of elastic-perfectly plastic spheres". Journal of Applied Mechanics, 64(2), p. 383-386.

[35] Gorham, D., and Kharaz, A., 2000. "The measurement of particle rebound characteristics". Powder Technology, 112(3), p. 193-202.

[36] Mueller, P., Antonyuk, S., Stasiak, M., Tomas, J., and Heinrich, S., 2011. "The normal and oblique impact of three types of wet granules". Granular Matter, 13(4), Jun.

[37] Lehman, R. The Mechanical Properties of Glass, Glass Engineering 150:312, Department of Ceramics and Materials Engineering, Rutgers University. University Lecture: http: / / glassproperties.com/references / MechPropHandouts.pdf.

[38] Aluminum 7178-T6 Data Sheet. [online] http : / / www . matweb . com / search / DataSheet . aspx ? MatGUID = $606 e 61$ c4801648c59d6d3215fa6824a5.

[39] Overview of Materials for High Carbon Steel. [online] http : / / www . matweb . com / search / datasheet . aspx ? matguid = ee25302df4b34404b21ad67f8a83e858.

[40] Hunter, S. C., 1957. "Energy absorbed by elastic waves during impact". Journal of the Mechanics and Physics of Solids, 23(5), pp. 165-171.

[41] Kruggel-Emden, H., Wirtz, S., and Scherer, V., 2008. "A study on tangential force laws applicable to the discrete element method (DEM) for materials with viscoelastic or plastic behavior". Chemical Engineering Science, 63(6).

[42] Maw, N., Barber, J., and Fawcett, J., 1976. "The oblique impact of elastic spheres". Wear, 38(1), p. 101-114. 